\title{
A!
}

This is an electronic reprint of the original article.

This reprint may differ from the original in pagination and typographic detail.

Saarikoski, Henri; Reimann, S.M.; Räsänen, Esa; Harju, Ari; Puska, Martti

\section{Stability of vortex structures in quantum dots}

Published in:

Physical Review B

DOI:

10.1103/PhysRevB.71.035421

Published: 26/01/2005

Document Version

Publisher's PDF, also known as Version of record

Please cite the original version:

Saarikoski, H., Reimann, S. M., Räsänen, E., Harju, A., \& Puska, M. (2005). Stability of vortex structures in quantum dots. Physical Review B, 71(3), 1-7. [035421]. https://doi.org/10.1103/PhysRevB.71.035421

This material is protected by copyright and other intellectual property rights, and duplication or sale of all or part of any of the repository collections is not permitted, except that material may be duplicated by you for your research use or educational purposes in electronic or print form. You must obtain permission for any other use. Electronic or print copies may not be offered, whether for sale or otherwise to anyone who is not an authorised user. 


\title{
Stability of vortex structures in quantum dots
}

\author{
H. Saarikoski, ${ }^{1}, *$ S. M. Reimann, ${ }^{2, \dagger}$ E. Räsänen, ${ }^{1}$ A. Harju, ${ }^{1}$ and M. J. Puska ${ }^{1}$ \\ ${ }^{1}$ Laboratory of Physics, Helsinki University of Technology, P.O. Box 1100, FIN-02015 HUT, Finland \\ ${ }^{2}$ Mathematical Physics, Lund Institute of Technology, SE-22100 Lund, Sweden \\ (Received 19 August 2004; revised manuscript received 29 October 2004; published 26 January 2005)
}

\begin{abstract}
We study the stability and structure of vortices emerging in two-dimensional quantum dots in high magnetic fields. Our results obtained with exact diagonalization and density-functional calculations show that vortex structures can be found in various confining potentials. In nonsymmetric external potentials we find offelectron vortices that are localized giving rise to charge deficiency or holes in the electron density with rotating currents around them. We discuss the role of quantum fluctuations and show that vortex formation is observable in the energetics of the system. Our findings suggest that vortices can be used to characterize the solutions in high magnetic fields, giving insight into the underlying internal structure of the electronic wave function.
\end{abstract}

DOI: 10.1103/PhysRevB.71.035421

PACS number(s): 73.21.La, 73.43.-f, 85.35.Be

\section{INTRODUCTION}

Vortices can occur in quantum systems which are set to rotate, for example by applying an external magnetic field or by mechanical rotation. ${ }^{1-3}$ Using the Gross-Pitaevskii meanfield approach, Butts and Rokhsar ${ }^{3}$ found that in a gas of rotating bosonic atoms which are weakly interacting by a repulsive force between them, vortices may form in a crystallike lattice, in much analogy to patterns that emerge in rotating superfluid helium. These vortex solutions appear as holes (vortex lines) in the particle densities, where each single zero of the Gross-Pitaevskii wave function corresponds to a unit vortex. With increasing angular momentum, the bosonic cloud develops a flat shape, with more and more vortices penetrating it.

The analysis of the electronic structure of twodimensional parabolic quantum $\operatorname{dots}^{4,5}$ (QD) in high magnetic fields has recently shown that vortices can appear also in fermion systems showing many similarities to the boson case. ${ }^{6,7}$ High-field solutions of the mean-field densityfunctional theory revealed zeros in the electron densities, with electron currents circulating around them, which were interpreted as vortex clusters. ${ }^{6}$ Many-body techniques could uncover vortex formation at high magnetic fields ${ }^{6,8}$ or at large rotation, ${ }^{7}$ giving credence to this interpretation. With increasing magnetic field or rotation, successive transitions between stable vortex configurations were found. ${ }^{6}$

Even though emergence of vortices inside QD's has been predicted by both mean-field and exact methods the interpretation of the effects of vortices needs careful analysis using both methods. As it is characteristic for mean-field theories, the self-consistent solutions of the Kohn-Sham equations for fermions, as well as the solutions of the Gross-Pitaevskii equation, being their bosonic equivalent, break the symmetry of the quantum state. For Bose-Einstein condensates under rotation, the Gross-Pitaevskii mean-field results were shown to emerge as the correct leading-order approximation to exact calculations. ${ }^{9}$ For Coulomb-interacting fermions, the density-functional approach suffers from the relatively crude approximations for the exchange-correlation energy, as well as from the problems in using a single-configuration wave function. ${ }^{10}$ The mean-field approach is, however, indispens- able since analytic solutions are out of reach for $N \geqslant 3$, and a numerically accurate direct diagonalization is also typically restricted to fairly small particle numbers.

The exact diagonalization studies of the vortices in fermionic systems have so far concentrated on parabolically confined QD's. In this case the rotational symmetry of the Hamiltonian implies rotational symmetry for the particle density in the laboratory frame of reference. Therefore, to study the appearance of vortices in those systems, a rotating frame, ${ }^{11}$ conditional wave functions, ${ }^{6}$ or correlation functions have to be examined. In contrast to the bosonic case, ${ }^{12}$ the pair correlations are not very informative for fermions due to the disturbing influence of the exchange hole. Instead, the vortex solutions have been pinpointed either in a perturbative approach, ${ }^{7}$ or using a conditional wave function which fixes $N-1$ electrons to their most probable positions, and the wave function of the $N$ th electron is calculated. ${ }^{6}$ The vortices in conditional wave functions can be seen as zeros associated with a phase change of integer multiple of $2 \pi$ for each path enclosing a zero. This method is not unproblematic either, since the vortices are not independent of the electron dynamics. The vortex locations in the conditional wave function depend on the positions of the fixed electron coordinates as well as on the choice of the probing electron.

The vortices in QD's have much in common with those in the fractional quantum Hall effect (FQHE). In FQHE the system can be approximated by Laughlin wave functions which attach additional vortex zeros at each electron. ${ }^{13}$ Both in finite QD's and infinite FQHE state the vortices carry magnetic flux quanta and they cause strong electron-electron correlations. Electronic structure calculations showed that in quantum dots ${ }^{6}$ and quantum dot molecules ${ }^{14}$ vortices were found to be bound on the electron positions, similarly to the Laughlin wave functions. A single vortex bound to an electron is Pauli vortex, because it is mandated by the exclusion principle. In general, the number of vortices on top of electron must be odd for fermions to have correct particle statistics. The calculations in QD's showed also another mechanism to cause strong electron-electron correlations: solutions were found with additional vortices moving between the electrons. ${ }^{6}$ These off-electron vortices give rise to rotating 
currents of charge and a charge deficiency down to zero electron density at the vortex centra.

In the FQHE the off-particle zeros are usually contrasted to on-particle zeros. ${ }^{15}$ The on-particle zeros are independent of electron coordinates except the coordinate of the electron to which the vortex is attached. On the other hand, the offparticle zeros of a given particle are not necessarily offparticle zeros of other particles. Graham et al. ${ }^{16}$ used this fact to conclude that off-particle zeros are not vortices in the real sense and therefore no charge deficiency is necessarily associated with them. Analogously to the FQHE case, the conditional wave functions indicate that the electrons in quantum dots see different positions for the off-particle vortices. In our calculations, however, we find evidence that this is a manifestation of zero-point motion of vortices which causes quantum fluctuations. Our results indicate formation of stable vortex structures where fluctuations slightly delocalize the vortices but, nevertheless, they can be clearly seen in the exact particle and current densities as rotating current of charge around density minima.

In this paper we show that in quantum dots only the offelectron zeros give rise to vortex structures in the electron and current densities. We do this by applying nonsymmetric confining potentials which leads to the localization of the vortices. In the rotationally symmetric potential vortices are not localized and they move as the electron coordinates are changed averaging out the effect of vortices on the particle and current densities. In rotationally nonsymmetric potentials, however, the lower symmetry should cause (at least partial) localization of vortices directly in the particle density. The breaking of the circular symmetry was used already by Manninen et al. in the study of Wigner localization of electrons in elliptical QD's using exact diagonalization (ED) techniques. ${ }^{17}$ Here we show that the same trick can be applied to vortices which become directly visible in the exact particle and current densities. We find that the Pauli vortices at the electron positions do not contribute to this effect. In our calculations even a small asymmetry in the confining potential is sufficient to cause partial localization of the additional vortices. These vortices can be treated as holelike quasiparticles which have zero-point motion and therefore they cannot be completely localized to a particular point in space. ${ }^{18}$ We compare the exact results to the mean field solutions and suggest that the results can be generalized to arbitrary geometries. We conclude that the vortex structures are stable and they can be used to classify the internal structure of the many-electron wave function.

\section{SYSTEM CHARACTERISTICS}

We consider $N$ electrons trapped by a confining potential $V_{c}$ and subject to a perpendicular, homogeneous magnetic field $\mathbf{B}=(0,0, B)$. The system is described by an (effectivemass) Hamiltonian

$$
H=\sum_{i=1}^{N}\left[\frac{\left(\mathbf{p}_{i}+e \mathbf{A}\right)^{2}}{2 m^{*}}+V_{\mathrm{c}}\left(\mathbf{r}_{i}\right)\right]+\frac{e^{2}}{4 \pi \epsilon} \quad \sum_{i<j} \frac{1}{\left|\mathbf{r}_{i}-\mathbf{r}_{j}\right|},
$$

where $\mathbf{A}$ is the vector potential of the magnetic field $B, m^{*}$ the effective electron mass, and $\epsilon$ is the dielectric constant of the medium. We apply the typical material parameters for GaAs, namely, $m^{*} / m_{e}=0.067$ and $\epsilon / \epsilon_{0}=12.4$. We give the energies and lengths in effective atomic units, i.e., in $\mathrm{Ha}^{*}$ $\simeq 11.86 \mathrm{meV}$ and in $a_{B}^{*} \simeq 9.79 \mathrm{~nm}$.

At high magnetic fields, after complete polarization of the QD, the exchange energy results in the formation of a stable and compact structure, the so called maximum density droplet (MDD). ${ }^{19}$ It is a finite-size precursor of the $\nu=1$ quantum Hall state which assigns one Pauli vortex at each electron position. The MDD state can be found in various QD geometries: in circularly confined QD's the electrons occupy successive angular momentum states on the lowest Landau level, which in the parabolic case leads to a relatively flat electron density. In rotationally nonsymmetric potential wells, instead, the MDD window can be deduced from the kinks in the chemical potentials. In addition, the magnetic field for the MDD formation can be accurately predicted from the number of flux quanta penetrating the QD. ${ }^{20}$

As the magnetic field is increased, the compact electron droplet is squeezed and eventually the repulsive interactions between the fermions cause the MDD to reconstruct. For parabolically-confined QD's, different scenarios of the reconstruction have been suggested. Chamon and $\mathrm{Wen}^{21}$ found a "stripe phase" where a lump of electrons separates from the MDD at a distance $\approx 2 \ell_{B}$, where $\ell_{B}=\sqrt{\hbar / e B}$ is the magnetic length. Goldmann and Renn introducted projected necklace states which they found to be lower in energy than the states found by Chamon and Wen. ${ }^{22}$ Geometrically unrestricted Hartree-Fock ${ }^{23}$ and CSDFT (Refs. 24 and 25) studies suggested that such edge reconstruction would occur with a modulated charge density wave along the edge. For a sufficiently small Zeeman gap, this polarized reconstruction may be preempted by edge spin textures. ${ }^{26,27}$

The ED shows instability with respect to addition of internal holes as discussed by Yang and MacDonald. ${ }^{28}$ These holes were recently reinterpreted as vortices in mean-field density-functional calculations. ${ }^{6}$ They were found also further away from the dot center where the electron density is low. Vortices behave often like classical (localized) particles in the mean-field approach and the vortices appear as rotating currents of charge with a zero in the particle density at the vortex centra. Several charge-density-wave states that mix different eigenstates can also be interpreted as solutions describing a transport of a vortex to the center of a QD. ${ }^{25}$ Formation of vortices causes usually a broken symmetry in the mean-field particle density, even when the Hamiltonian is azimuthally symmetric.

Tavernier et al. ${ }^{8}$ studied distribution of zeros in the exact many-body wave function of systems containing up to 4 electrons. They compared the results with the rotatingelectron-molecule (REM) wave functions. ${ }^{29}$ In the regime where the effect of the external confining potential can be neglected, the rotating electron molecule model can provide an intuitive description of the Wigner-localized electrons. Tavernier and co-workers ${ }^{8}$ found out, however, that the REM model is unable to predict the clustering of vortices near electrons. 


\section{NUMERICAL RESULTS}

\section{A. Mean-field description of vortex solutions}

In order to solve the many-body Schrödinger equation corresponding to the Hamiltonian (1), we first work in the mean-field picture and apply the spin-density-functional theory (SDFT). For the self-consistent solution of the KohnSham equations we employ a real-space scheme, ${ }^{30}$ where the external confining potential $V_{\mathrm{c}}$ can be arbitrarily chosen without symmetry restrictions. The exchange-correlation effects are taken into account using the local spin-density approximation (LSDA). ${ }^{31}$ At high magnetic fields, the effect of currents in the exchange-correlation potentials becomes nonnegligible, and the current-spin-density-functional theory (CSDFT)(Ref. 32) gives a slightly better approximation to the ground state energy. ${ }^{33}$ The CSDFT is computationally more demanding than the SDFT but according to our test calculations qualitatively similar vortex structures were found to emerge in both formalisms. We apply the SDFT throughout this paper, since we found in these tests that it captures all the essential physics of these systems at much lower computational work.

The confinement is chosen to be a two-dimensional harmonic oscillator potential with elliptic deformation, defined as

$$
V_{c}(\mathbf{r})=\frac{1}{2} \hbar \omega_{0}^{2}\left(\delta x^{2}+\frac{1}{\delta} y^{2}\right),
$$

where $x$ and $y$ are the major axes of the ellipse, $\hbar \omega$ is the confinement strength, and $\delta$ is the eccentricity. For comparison, we also apply a rectangular hard-wall confinement, defined in Ref. 20. The parameter describing the deformation of the confining potential in this case is the side-length ratio $\beta=L_{x} / L_{y}$.

In the post-MDD domain, the SDFT predicts the formation of vortices inside the QD's. This is visualized in Fig. 1 showing solutions containing up to three vortices in elliptic $(\mathrm{a}-\mathrm{d})$ and rectangular $(\mathrm{e}-\mathrm{h})$ six-electron QD's. The eccentricity $\delta$ and the side-length ratio $\beta$ have been set to 2 in these systems, respectively. As the vortices repel each other, the MDD states shown in Figs. 1(a) and 1(e) reconstruct into states that enclose a linear vortex pattern along the longest major axis. There is a remarkable qualitative similarity in the high magnetic field behavior of these systems. However, a linear vortex cluster requires a rather large eccentricity (sidelength ratio) of the QD. In the elliptic case with a confinement strength of $\hbar \omega=0.5 \mathrm{Ha}^{*}$, the triple-vortex configuration changes from triangular to linear when $\delta$ is increased to about 1.4.

Figure 1 shows currents induced by the magnetic field. The current is flowing clockwise around the vortices and anticlockwise on the edges of the dot. The reversal of the current near the vortex core is due to inner circulation of the electrons. ${ }^{38}$ The number of vortices in the QD increases with the magnetic field, and the current loops of the vortices start to overlap. This causes formation of giant current loops which comprise several vortices.

Figure 2 shows the chemical potentials, $\mu(N)=E(N)$ $-E(N-1)$, of a rectangular $(\beta=2)$ QD containing $N$
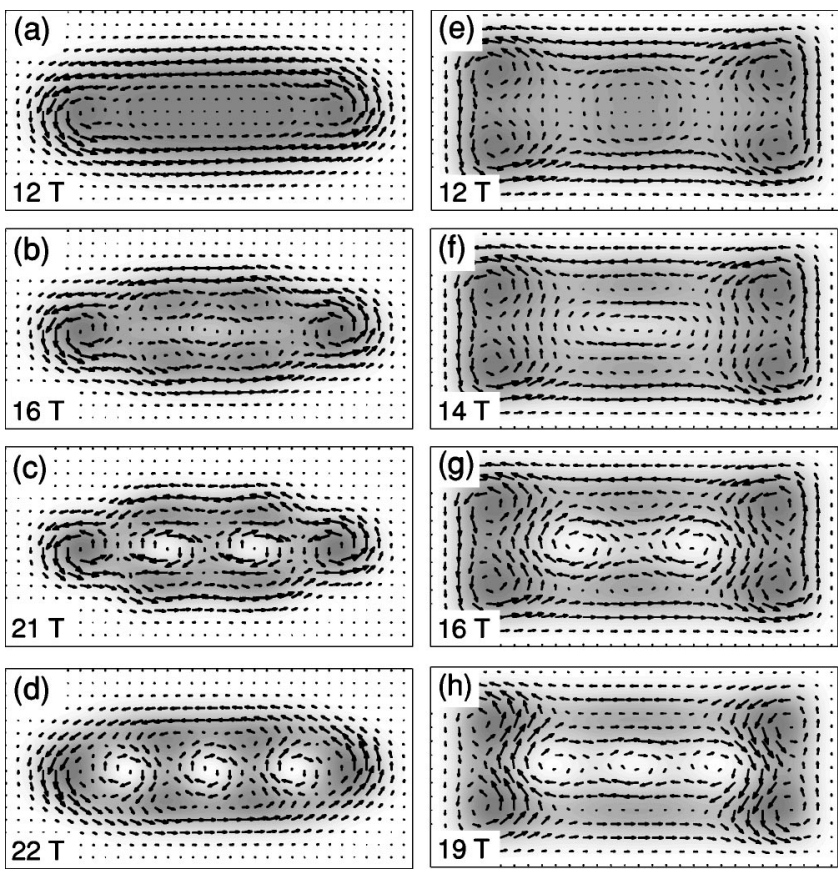

FIG. 1. Spin-density-functional-theory (SDFT) electron densities and currents in an elliptic (a-d) and rectangular (e-h) sixelectron quantum dot in different magnetic fields. The potential parameters in effective a.u. are $(\hbar \omega, \delta)=(0.5,2)$ and $\left(L_{x}, L_{y}\right)$ $=(2 \sqrt{2 \pi}, \sqrt{2 \pi})$ in elliptic and rectangular dots, respectively. The increasing of the magnetic field leads to a formation of a vortex pattern beyond the maximum density droplet (MDD) solution ( $B$ $=12 \mathrm{~T})$ in both geometries.

$=7, \ldots, 16$ electrons. Interestingly, the regime beyond the MDD is characterized by periodic oscillations in $\mu$ as a function of the magnetic field $B$. The peak positions in the oscillations match with the transitions between adjacent vortex states and mark the emergence of additional vortices one-byone in the QD, presented for $N=6$ in Figs. 1(f) $-1(\mathrm{~h})$. The oscillations get stronger as the number of electrons increases. The origin of the oscillations lies in the large reduction of the

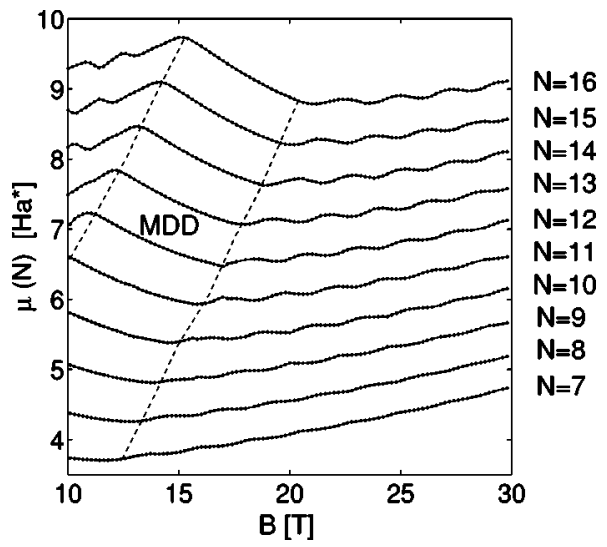

FIG. 2. Chemical potentials for $N$-electron rectangular $(\beta=2)$ quantum dots as a function of the magnetic field. The dot is expected to be fully spin-polarized. After the MDD window there are oscillations in the chemical potential which matches with the appearing of additional vortices one-by-one into the quantum dot. 


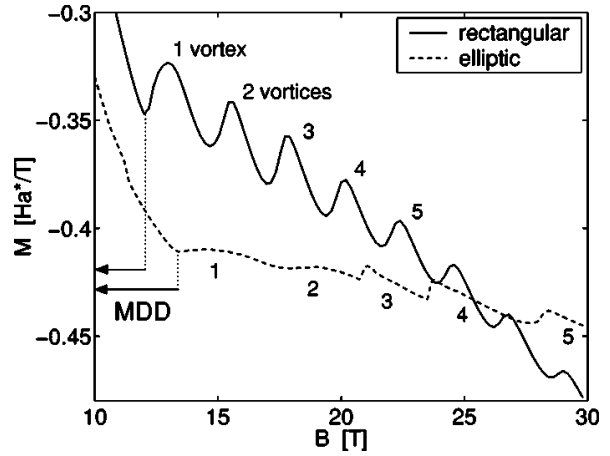

FIG. 3. Magnetization of a rectangular (solid line) and elliptical (dashed line) six-electron quantum dot as a function of the magnetic field. The ellipse eccentricity $\delta$ and the rectangle side-length ratio $\beta$ are both set to 2 . The numbers in the figure denote the correponding number of vortex holes in the electronic structure.

Coulomb energy in connection with the vortex formation and the coexistent pronounced localization of the electrons. The oscillations are also visible in the total magnetization, $M=$ $-\partial E_{\mathrm{tot}} / \partial B$, as shown in Fig. 4 in Ref. 20. The magnetization in rectangular and elliptic six-electron QD's are compared in Fig. 3.

In elliptic QD's the oscillations are weaker and less regular than in their rectangular counterparts where the size of the dot is a constant. The soft-wall confinement makes the dot more flexible in minimizing the total energy. These results are in accord with the exact diagonalization results by Goldmann and Renn, ${ }^{22}$ who calculated the chemical potentials of QD's in parabolic and nonparabolic "coffee-cup" shaped confinements. The latter confinement has a hard wall similarly to our rectangular potential well. Goldmann and Renn found kinks in the chemical potentials and the kink sizes increase with the electron number. Moreover, the kinks in the nonparabolically confined systems were found to be much larger than in the parabolically confined systems.

The results above suggest that the vortex formation is a considerable energetic effect that could be detected in appropriate experiments. Oosterkamp et al. ${ }^{34}$ measured the Coulomb oscillations peaks (chemical potentials) in transport experiments for vertical QD's and observed additional phase transitions beyond the MDD. They found oscillations in chemical potentials and the amplitude of these oscillations increased with the electron number. This data is consistent with our calculations and could indicate vortex formation in quantum dots. A direct interpretation of their result is, however, difficult due to the unknown shape of the QD sample and its eventual sensitivity to disturbance in the experiment. Moreover, the experimental oscillations may also indicate other phenomena, such as the formation of a spin texture, for example. ${ }^{26}$

Conditional wave functions can be introduced not only for the analysis of the exact many-body wave function, as described in Ref. 6, but they are also useful to analyze the SDFT results. We use an auxiliary single-determinant function of the Kohn-Sham orbitals which emulates the exact conditional wave function..$^{25}$ This allows a study of the SDFT solutions where the electron density may have several minima, but the vortices are not directly localized to fixed positions. This may be due to the mixing of several eigenstates as shown in Ref. 25. For instance, the electron densities for the SDFT states at $18 \mathrm{~T}$ and $21 \mathrm{~T}$ show no density zeros. However, the corresponding conditional singledeterminant functions show vortices near the fixed electron ring (see Fig. 4).

In this picture Pauli vortices can be seen on the fixed electrons, and additional vortices are found between the electrons.

The electron densities of the elliptical $\delta=2$ dot in Fig. 4 show localization of both electrons and vortices. The electron localization results in six density maxima which is consistent with earlier SDFT calculations of elliptically confined QD's. ${ }^{17}$ The additional vortices give rise to charge deficiency in the electron density as seen in the right panel of Fig. 4. According to the SDFT results, the intensity of the vortex localization strongly depends on the magnetic field. In the above example, the localization of vortices is partial with pronounced minima in the electron density in solutions of up to two vortices. As the magnetic field is increased further, the localization becomes complete i.e. the electron density vanishes at the vortex core. This reflects the decrease of the magnetic length $\ell_{B}$.

The results indicate that only the additional vortices between the electrons have a charge deficiency associated with them. Conditional wave functions ${ }^{6}$ and the total electron den-

\section{(a) $18 \mathrm{~T}$}

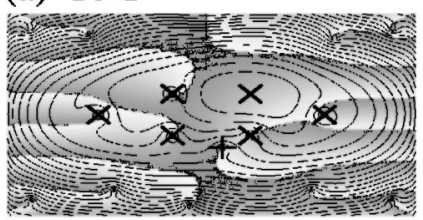

(b) $21 \mathrm{~T}$

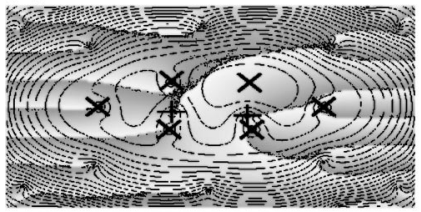

(c) $22 \mathrm{~T}$
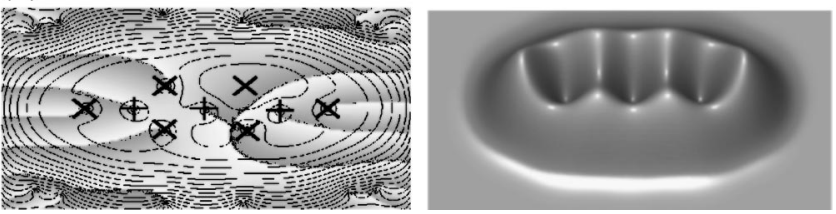

FIG. 4. SDFT solutions of six-electron, elliptically $(\delta=2)$ confined quantum dots at different magnetic fields. The confinement strength is set to $\hbar \omega=0.5 \mathrm{Ha}^{*}$. Left panel: Conditional singledeterminant wave functions of the Kohn-Sham states. The fixed electrons are marked with crosses and the probing electron is the rightmost electron at the top. The contours show the logarithmic electron density of the probe electron and the grey-scale show the phase of the wave function. The phase changes from $\pi$ to $-\pi$ at the lines where shadowing changes from the darkest grey to white. The vortices that cause charge deficiency in the center of the dot are marked with + signs. Right panel: Electron densities show vortex holes and partial localization of the electrons forming six density maxima. 
sities calculated with the SDFT show that there are vortices also further away from the dot center. In this region the electron density is usually a tiny fraction of the maximum electron density. The quantum fluctuations smooth out the effect of these external vortices and they cannot be directly observed in the exact particle density, even though the rotational symmetry is broken.

\section{B. Exact diagonalization for an elliptic dot}

We compare now the above results obtained within SDFT to those of a direct numerical diagonalization of the manybody Hamiltonian matrix. We hereby focus on small particle numbers and magnetic fields high enough such that the description is mostly restricted to what in the isotropic case would correspond to the lowest Landau level. In order to display the internal structure of the many-body wave function, we break the spherical symmetry of the dot by applying the elliptical confining potential [Eq. (2)]. We assume full polarization of the electron droplet, and neglect the Zeeman energy.

For the deformed case, $\delta \neq 1$, where the total angular momentum is not any more a good quantum number (while, however, we still have good parity), the most appropriate and efficient basis set spanning the Fock space is formed by the eigenstates to the single-particle part of the Hamiltonian (1). These must be calculated numerically. We determine the $\mathcal{M}$ lowest ones by directly diagonalizing the single-particle part of the Hamiltonian in a basis consisting of a sufficient number of corresponding Fock-Darwin ${ }^{35}$ states at $\delta=1$, which are known analytically. Once the single-particle basis is at disposal, the Fock states are generated by sampling over all possibilities to set $N$ particles on these $\mathcal{M}$ states. From this sampling, only those Fock states with defined parity and configuration energy ${ }^{36}$ less than a defined cut-off energy, are chosen for diagonalization. The cut-off energy was adjusted to restrict the number of Fock states (i.e., the matrix dimension) to be less than about 50000 . We limit the singleparticle basis dimension to $\mathcal{M} \leqslant 44$ and use numerical integration for calculating the Coulomb matrix elements. The many-body Hamiltonian (1) is then diagonalized in the obtained subspace. Densities $\langle\hat{n}(\mathbf{r})\rangle$ and real currents $\langle\hat{\mathbf{j}}(\mathbf{r})\rangle$ are finally calculated in order to compare the broken-symmetry solutions of the ED directly to the mean-field results. Here,

$$
\hat{n}(\mathbf{r})=\sum_{i} \delta\left(\mathbf{r}-\mathbf{r}_{i}\right)
$$

is the density operator. The real current is obtained by taking the expectation value of

$$
\hat{\mathbf{j}}(\mathbf{r})=\hat{\mathbf{j}}_{p}(\mathbf{r})+\frac{e}{m^{*}} \mathbf{A}(\mathbf{r}) \hat{n}(\mathbf{r}),
$$

where

$$
\hat{\mathbf{j}}_{p}(\mathbf{r})=\sum_{i} \frac{-i \hbar}{2 m^{*}}\left[\delta\left(\mathbf{r}-\mathbf{r}_{i}\right) \nabla_{i}+\nabla_{i} \delta\left(\mathbf{r}-\mathbf{r}_{i}\right)\right]
$$

is the paramagnetic current operator.

We should note at this point that for obtaining an accurate description of the total energy of the system, using only 44

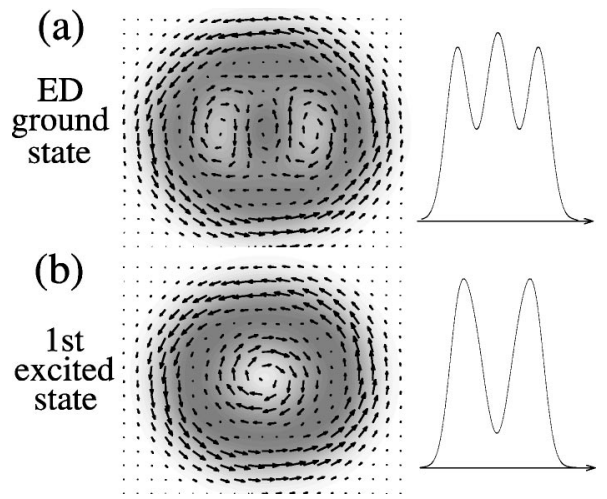

FIG. 5. Electron densities (grey scale) and current densities (arrows) of exact diagonalization (ED) solutions for an elliptical quantum dot with $\delta=1.1$. The number of electrons is 6 . (a) The ground state with a two-vortex structure. (b) The first excited state with a single vortex at the origin. The right column shows the corresponding electron densities at the longest major axis of the ellipse. The confinement strength is $\hbar \omega=0.5 \mathrm{Ha}^{*}$ and the magnetic field is $B$ $=13.4 \mathrm{~T}$.

lowest single-particle states is not sufficient for a full convergence of the total energy. However, the relative energy differences and the geometrical structure of the electron and current densities of the ground-state and lowest-lying states were converged within this basis set. Since our aim is the comparison of the broken-symmetry many-body with the mean-field solutions, rather than a detailed discussion of energy spectra and excitation energies, this truncation appeared reasonable.

Figure 5 shows the electron and current densities, $\langle\hat{n}(\mathbf{r})\rangle$ and $\langle\hat{\mathbf{j}}(\mathbf{r})\rangle$, of the ground state and first excited state for an elliptic dot with eccentricity $\delta=1.1$ and magnetic field 13.4 T. Both states have parity $\pi=-1$, and the first excited state is separated from the ground state by only $7.7 \mathrm{mHa}$. The ground state shows a vortex pattern around two minima in the density and its total angular momentum is $L=-25.4 \hbar$. The leading single-particle configuration ${ }^{7}$ of the Fock state has the form $|1001111100 \ldots\rangle$, with amplitude $\left|c_{\mathcal{L}}\right|^{2}=0.4$. The first excited state shows a pronounced single vortex at the dot center, with the current circulating clockwise around the origin. The hole shows the characteristic cone shape for a vortex and appears nearly localized at the dot center. The leading configuration of the Fock states is $|011111100 \ldots\rangle$ with amplitude $\left|c_{\mathcal{L}}\right|^{2}=0.7$. The second excited state has parity $\pi=+1$ and is separated by a gap of $53 \mathrm{mHa}^{*}$ from the ground state. It has angular momentum $L=-20.4 \hbar$ and no clear vortex structure.

Figure 6 shows the densities and density contours for the cases $\delta=1.1$ (a) and $\delta=1.2$ (b) at an increased magnetic field of $17 \mathrm{~T}$. Both states have parity $\pi=-1$ and angular momentum $L=-26.7 \hbar$ (a) and $L=-28.5 \hbar$ (b). In both cases, the current circulates around fairly pronounced minima in the charge density, representing two vortices at the dot center. When the eccentricity increases from $\delta=1.1$ (a) to $\delta=1.2(\mathrm{~b})$, the electrons begin to arrange themselves in the form of a Wigner molecule with counterclockwise rotation of the current around the maxima of the electron density, and clock- 
(a) $\delta=1.1 \quad \mathrm{ED}$
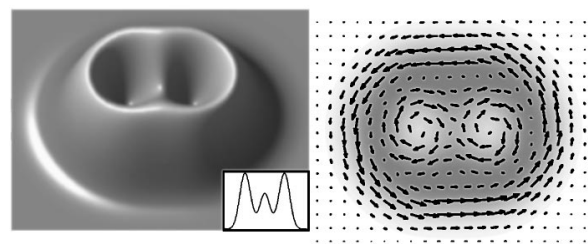

(b) $\delta=1.2 \quad \mathrm{ED}$
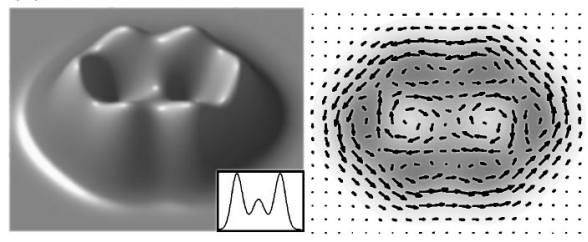

(c) $\delta=1.2$ SDFT

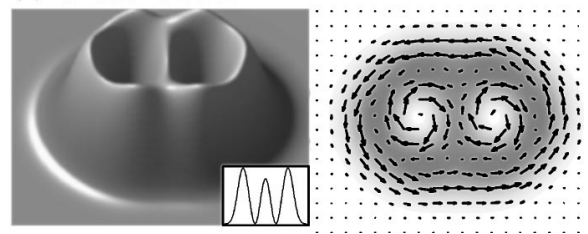

FIG. 6. Electron densities (gray scale) and current densities (arrows) for two-vortex solutions in elliptical QD's. The number of electrons is 6. (a) Exact diagonalization (ED) result at $\delta=1.1$. (b) ED result at $\delta=1.2$. (c) SDFT result at $\delta=1.2$. The inset shows the corresponding electron densities at the longest major axis of the ellipse. The vortices are localized in the SDFT solution, and the ED solution shows slight delocalization due to quantum fluctuations. The confinement strength is $\hbar \omega=0.5 \mathrm{Ha}^{*}$ and the magnetic field is $B=17 \mathrm{~T}$

wise rotation around the vortices (i.e., the minima in the density), as it was also observed at $\delta=1.1$. The reversal of the current near the vortex core is consistent with the SDFT results.

It is important to note here that the ground state in both cases is a state with $\pi=+1$, which for (a) is $34 \mathrm{mHa}^{*}$ and for (b) is $7.6 \mathrm{mHa}^{*}$ lower in energy. No states were found in between these two lowest states with $\pi=+1$ and $\pi=-1$, i.e. both states shown in (a) and (b) appeared as first excited states, respectively. The fact that the energetic sequence of the states changes with increasing field is, however, not surprising: It is well known ${ }^{5}$ from the ED studies of circularly symmetric QD's, that the overall structure of the spectrum is independent of the magnetic field, the role of which is mainly to tilt the spectrum so that the minimum energy is at a different state (see Manninen et al., ${ }^{17}$ and Maksym and Chakraborty $^{37}$ ).

The above results from the ED are compared with the mean-field calculation in Fig. 6(c) for $\delta=1.2$. The results appear fairly similar. The largest difference naturally appears due to the presence of quantum fluctuations which destroy the complete localization of the vortices, i.e. the exact density at the vortex center still is about one-third of the maximum density, while in the mean-field result the density at the vortex center is reduced to zero.

Increasing the eccentricity still further, the localization of electrons leads to the formation of a charge-density-wavelike crystal. Vortices in between the classical electron positions (a) $B=11 \mathrm{~T}$
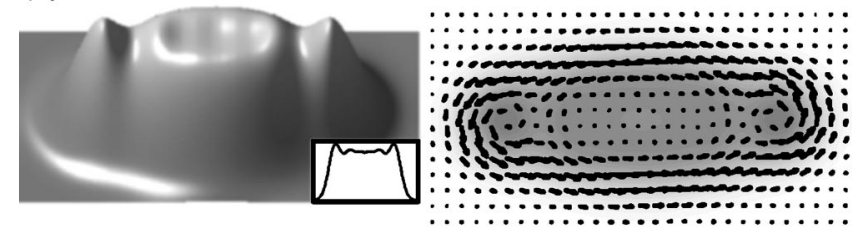

(b) $B=17 \mathrm{~T}$
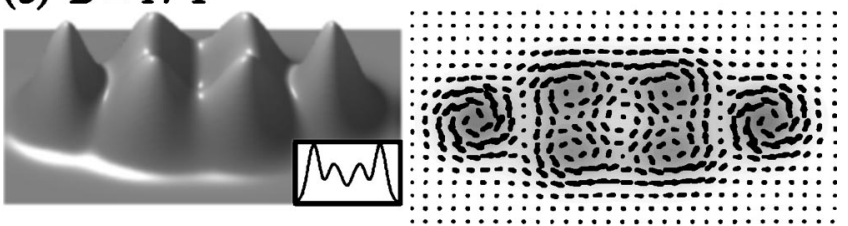

(c) $B=22 T$

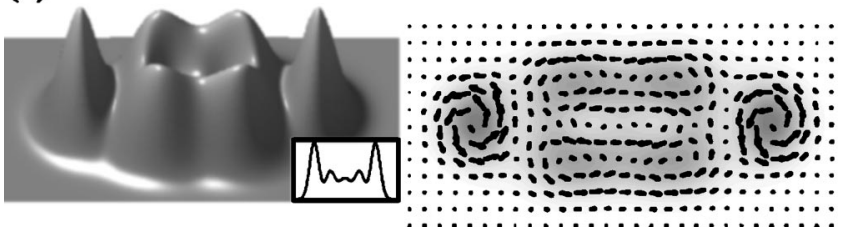

FIG. 7. Electron densities (grey scale) and current densities (arrows) of ED solutions for elliptical quantum dots with $\delta=2$. The magnetic field is (a) $17 \mathrm{~T}$, (b) $21 \mathrm{~T}$, and (c) $22 \mathrm{~T}$. The inset shows the corresponding electron densities at the longest major axis of the ellipse. The number of electrons is 6 and the confinement strength is $\hbar \omega=0.5 \mathrm{Ha}^{*}$.

become even more apparent. Figure 7 shows the ED electron densities (grey scale) and current densities (arrows) for larger eccentricity $\delta=2$. The magnetic field is (a) $11 \mathrm{~T}$, (b) $17 \mathrm{~T}$, and (c) $22 \mathrm{~T}$, and $\pi=-1$ in all cases. (The $\pi=+1$ states are slightly higher in energy.) The solution at $11 \mathrm{~T}$ shows the MDD, and vortices form at higher magnetic fields. These solutions can be compared to the SDFT solutions in Fig. 1. There is again a qualitative agreement, but now the ED shows stronger localization of electrons at both ends of the dot. While now the Fock states show much mixing and a clear dominance of a few single configurations could not be observed, the general trend is very similar to the SDFT result shown above in Fig. 1.

\section{SUMMARY}

We have found that in noncircular confinements the vortices can be directly seen in the exact diagonalization electron densities as holes or charge deficiency around which the current is circulating. The vortex structures are stable against fluctuations and even a slight asymmetry in the confining potential cause the vortices to show up in the exact manybody electron density as density minima. However, due to zero-point motion of vortices there are no zeros in the electron density. The spin-density-functional calculations are in accord with these results. They predict analogous vortex formation also in rectangular hard-wall quantum dots suggesting that the results can be generalized to a wide variety of geometries. The chemical potentials of the rectangular dot show features in the energetics that could be directly compared to the experiments. 
In the light of these results it is justified to speak of vortices as real quasiparticles which might have observable effects in the measurements. Vortices are not independent of the electron dynamics, but they can be used to characterize the solutions in high magnetic fields which gives insight of the underlying internal structure of the electronic wave function. Prediction of vortex formation in hard-wall potentials gives also credence to the assumption that the vortices are robust and largely independent of the chosen geometry of the system. Therefore they seem to be universal features of the physics of the two-dimensional interacting fermion systems in strong magnetic fields above the maximum density droplet formation.

To conclude, we briefly compare these results to bosonic systems, where vortex formation in rotating Bose-Einstein condensates has been much discussed both theoretically and experimentally. There are apparent similarities between the bosonic and fermionic case. In the composite fermion model bosons can be turned into fermions (and the other way around) by attaching fictious magnetic fluxes on top of electrons. Vortex formation appears as a universal phenomenon of quasi-two-dimensional quantum systems suggesting a synthetic theoretical rationale behind the phenomena. ${ }^{39}$

\section{ACKNOWLEDGMENTS}

Special thanks go to Matti Koskinen for his help with the exact diagonalization code. We also thank Matti Manninen, Ben Mottelson, and Maria Toreblad for fruitful discussions. This work has been supported by the Academy of Finland through the Center of Excellence Program (2000-2005), the Swedish Foundation for Strategic Research (SSF) and the Swedish Research Council (VR).
*Electronic address: henri.saarikoski@hut.fi

†Electronic address: reimann@matfys.lth.se

${ }^{1}$ P. G. de Gennes, Superconductivity of Metals and Alloys (Benjamin, New York, 1966).

${ }^{2}$ E. J. Yarmchuk, M. J. V. Gordon, and R. E. Packard, Phys. Rev. Lett. 43, 214 (1979).

${ }^{3}$ D. A. Butts and D. S. Rokhsar, Nature (London) 397, 327 (1999).

${ }^{4}$ L. Jacak, P. Hawrylak, and A. Wójs, Quantum Dots (Springer, Berlin, 1998).

${ }^{5}$ S. M. Reimann and M. Manninen, Rev. Mod. Phys. 74, 1283 (2002).

${ }^{6}$ H. Saarikoski, A. Harju, M. J. Puska, and R. M. Nieminen, Phys. Rev. Lett. 93, 116802 (2004).

${ }^{7}$ M. Toreblad, M. Borgh, M. Koskinen, M. Manninen, and S. M. Reimann, Phys. Rev. Lett. 93, 090407 (2004).

${ }^{8}$ M. B. Tavernier, E. Anisimovas, and F. M. Peeters, Phys. Rev. B 70, 155321 (2004).

${ }^{9}$ A. D. Jackson, G. M. Kavoulakis, B. Mottelson, and S. M. Reimann, Phys. Rev. Lett. 86, 945 (2001).

${ }^{10}$ A. Harju, E. Räsänen, H. Saarikoski, M. J. Puska, R. M. Nieminen, and K. Niemelä, Phys. Rev. B 69, 153101 (2004).

${ }^{11}$ P. A. Maksym, Phys. Rev. B 53, 10871 (1996).

${ }^{12}$ G. M. Kavoulakis, S. M. Reimann, and B. Mottelson, Phys. Rev. Lett. 89, 079403 (2002).

${ }^{13}$ T. Chakraborty and P. Pietiläinen, The Quantum Hall Effects: Fractional and Integral (Springer, Berlin, 1995).

${ }^{14}$ A. Harju, S. Siljamäki, and R. M. Nieminen, Phys. Rev. Lett. 88, 226804 (2002).

${ }^{15}$ Ganpathy Murthy and R. Shankar, Rev. Mod. Phys. 75, 1101 (2003).

${ }^{16}$ Kenneth L. Graham, Sudhansu S. Mandal, and Jainendra K. Jain, Phys. Rev. B 67, 235302 (2003).

${ }^{17}$ M. Manninen, M. Koskinen, S. M. Reimann, and B. Mottelson, Eur. Phys. J. D 16, 381 (2001).

${ }^{18}$ M. Manninen, S. M. Reimann, M. Koskinen, Y. Yu, and M. Toreblad, cond-mat/0410622 (unpublished).

${ }^{19}$ A. H. MacDonald, S.-R. Eric Yang, and M. D. Johnson, Aust. J.
Phys. 46, 345 (1993).

${ }^{20}$ E. Räsänen, A. Harju, M. J. Puska, and R. M. Nieminen, Phys. Rev. B 69, 165309 (2004).

${ }^{21}$ C. de Chamon and X. G. Wen, Phys. Rev. B 49, 8227 (1994).

${ }^{22}$ E. Goldmann and S. R. Renn, Phys. Rev. B 60, 16611 (1999).

${ }^{23}$ H.-M. Müller and S. E. Koonin, Phys. Rev. B 54, 14532 (1996).

${ }^{24}$ S. M. Reimann, M. Koskinen, M. Manninen, and B. R. Mottelson, Phys. Rev. Lett. 83, 3270 (1999).

${ }^{25}$ H. Saarikoski, A. Harju, M. J. Puska, and R. M. Nieminen, condmat/0404704, Physica E (to be published).

${ }^{26}$ A. Karlhede, S. A. Kivelson, K. Lejnell, and S. L. Sondhi, Phys. Rev. Lett. 77, 2061 (1996).

${ }^{27}$ S. Siljamäki, A. Harju, R. M. Nieminen, V. A. Sverdlov, and P. Hyvönen, Phys. Rev. B 65, 121306(R) (2002).

${ }^{28}$ S.-R. Eric Yang and A. H. MacDonald, Phys. Rev. B 66, 041304(R) (2002).

${ }^{29}$ C. Yannouleas and U. Landman, Phys. Rev. B 66, 115315 (2002); 68, 035326 (2003).

${ }^{30}$ H. Saarikoski, E. Räsänen, S. Siljamäki, A. Harju, M. J. Puska, and R. M. Nieminen, Eur. Phys. J. B 26, 241 (2002).

${ }^{31}$ C. Attaccalite, S. Moroni, P. Gori-Giorgi, and G. B. Bachelet, Phys. Rev. Lett. 88, 256601 (2002).

${ }^{32}$ G. Vignale and M. Rasolt, Phys. Rev. B 37, 10685 (1988).

${ }^{33}$ H. Saarikoski, E. Räsänen, S. Siljamäki, A. Harju, M. J. Puska, and R. M. Nieminen, Phys. Rev. B 67, 205327 (2003).

${ }^{34}$ T. H. Oosterkamp, J. W. Janssen, L. P. Kouwenhoven, D. G. Austing, T. Honda, and S. Tarucha, Phys. Rev. Lett. 82, 2931 (1999).

${ }^{35}$ V. Fock, Z. Phys. 47, 446 (1928); C. G. Darwin, Proc. Cambridge Philos. Soc. 27, 86 (1930).

${ }^{36}$ S. M. Reimann, M. Koskinen, and M. Manninen, Phys. Rev. B 62, 8108 (2000).

${ }^{37}$ P. A. Maksym and T. Chakraborty, Phys. Rev. Lett. 65, 108 (1992); P. A. Maksym, Phys. Rev. B 53, 10871 (1996).

${ }^{38}$ C. S. Lent, Phys. Rev. B 43, 4179 (1991).

${ }^{39}$ H. Saarikoski et al. (unpublished). 